\title{
Música y cine documental. Un diálogo entre Errol Morris y Philip Glass: The Thin Blue Line (1988)
}

\section{Music and documentary films. A dialogue between Errol Morris and Philip Glass: The Thin Blue Line (1988)}

\author{
Pablo Vegas Fernández \\ Universidad de Oviedo, España \\ fernandezvegaspablo@gmail.com
}

\begin{abstract}
Resumen:
The Thin Blue Line es una de las obras fundamentales de la filmografía de Errol Morris. En este documental se consigue desvelar la inocencia de Randall Adams, acusado por el asesinato de un agente de policía en 1976. Sin embargo, los estudios sobre este film no se han detenido en la consideración de la música compuesta por Philip Glass: ésta juega un papel determinante en la construcción de la inocencia de Adams, así como en el descubrimiento de la corrupción de la justicia estadounidense. De este modo, los reenactments utilizados ampliamente por el director a lo largo del documental dialogarían continuamente con la música de Glass, construyendo entre ambos -tanto en clave irónica, como a través de la repetición- dicha inocencia. Partiendo de estudios sobre el cine documental, como los publicados por Bill Nichols $(2013,2016)$ o Jonathan Kahana (2016), y aquellos sobre musicología y música en el cine documental, como el editado por Holly Rogers (2016) o Tristan Evans (2015), este artículo trata de establecer relaciones entre los procedimientos de Morris y Glass, quienes a través del dialogo entre reenactments y los presupuestos del minimalismo musical consiguen evidenciar la inocencia de Randall Adams.
\end{abstract}

\begin{abstract}
:
The Thin Blue Line is one of the key works on Errol Morris' filmography. This documentary achieved the uncover of Randall Adams' innocence, who had been charged of police officer murder in 1976. Nevertheless, studies on this film hasn't focused yet on its music, composed by Philip Glass: it plays a major role as a constructor of Adams' innocence, as well as it takes part in revealing corruption of U.S. justice. Reenactments used by Errol Morris and music by Glass dialogue along the documentary, in an ironical way as well as through repetition. Starting from documentary film essays as those published by Bill Nichols $(2013,2016)$ or Jonathan Kahana (2016), and those concerning musicology as well as music in documentary film like Holly Rogers (2016) or Tristan Evans (2015), this article aims to stablish bounds between Morris and Glass' creative methods: through a dialogue between reenactments and musical minimalism's hypothesis they achieve to prove Randall Adams' innocence.
\end{abstract}

Palabras clave: música y cine documental; reenactments; minimalismo; interdisciplinaridad.

Keywords: Music and Documentary Films, Reenactments, Minimalism, Interdisciplinarity. 


\section{Introducción}

The Thin Blue Line surge a raíz de una investigación de Errol Morris en torno a la figura del Dr. James Grigson -apodado "Dr. Death"- un psiquiatra de Dallas que durante más de una década había testificado por el enjuiciamiento de casos correspondientes al corredor de la muerte (Resha, 2015, pp. 50-52). La labor de este doctor consistía en ofrecer valoraciones sobre la posible vuelta a la delincuencia de los acusados sirviéndose de unos exámenes orales o reuniones que no solían durar más de una hora ${ }^{1}$. Tras haber entrevistado a varios acusados que habían sido objeto de las predicciones de Grigson, Morris conoce a Randall Adams, condenado por el asesinato de un agente de policía el 29 de noviembre de 1979 en Dallas, Texas. Tras oír el testimonio de Adams, el director se convence de su inocencia y comienza a obsesionarse con su caso, desplazando finalmente el foco de su nuevo documental del "Dr. Death" al caso de Adams.

A lo largo del documental Morris desempeñará un doble rol de cineasta/detective, oficio este último que había realizado con anterioridad: poco después de la muerte de su hermano y desesperanzado en cuanto a la financiación de un nuevo proyecto cinematográfico, acepta un trabajo como detective privado investigando fraudes en el ámbito de Wall Street (Algar, 1989; Curry, 1995, p. 49; Millard, 2014, p. 65). Esta antigua ocupación se complementará con la búsqueda de la 'verdad' que Morris realizará a lo largo de su carrera, siendo The Thin Blue Line un óptimo ejemplo para demostrar la forma en que el director saca a la luz una evidencia: la injusta condena a un inocente.

Por otro lado, atendiendo a los aspectos musicales, hemos de considerar la participación de Philip Glass en la composición de música para documentales con anterioridad. Si bien el propio Glass afirma que nunca había querido componer música para cine, el director Godfrey Reggio consiguió convencerlo tras la proyección de unos fragmentos de Koyaanisqatsi (1983), documental que sería el punto de partida de Glass en la composición de música para cine (Glass, 2015, pp. 397-401). Asimismo, el compositor

\footnotetext{
${ }^{1}$ En el caso de Randall Adams, acusado en The Thin Blue Line, éste afirma haber pasado con el psiquiatra no más de unos 15 o 20 minutos.
} 
establece la condición de Errol Morris como uno de sus directores predilectos con los que compartir proyecto ${ }^{2}$, reconociendo su brillantez al "haber redefinido la relación del espectador con la temática de la película" (Glass, 2015, p. 424).

Gran parte del material empleado por Philip Glass para The Thin Blue Line guarda una estrecha relación con Metamorphosis (Glass, 2006 [1991]), obra publicada en el álbum de 1989 Solo Piano3. Así, se puede afirmar que la segunda pieza de Metamorphosis fue compuesta expresamente para este documental (Evans, 2015, p. 109) y que tanto la primera como la quinta se basan en material de The Thin Blue Line, tal y como afirma Jody Dalton en las notas a la grabación Solo Piano. Asimismo, este material será reutilizado en Las horas (The Hours, Stephen Daldry, 2002), enfatizando uno de los puntos clave de la narración -el suicidio de uno de los personajes- con el pasaje en tresillos de la segunda de las Metamorphosis (véase Ejemplo 1). No obstante, como veremos, en el caso de The Thin Blue Line este pasaje se usa de forma ligeramente distinta, trasladando este clímax musical al final del documental. No es éste el único caso en el que hay una relación entre obras de Glass y su reutilización en el cine, pudiendo mencionar, por ejemplo, la analogía entre las obras In the Upper Room (1986) y String Quartet No. 2 'Company' (1984) con el documental The Fog of War (2002) también dirigido por Errol Morris.

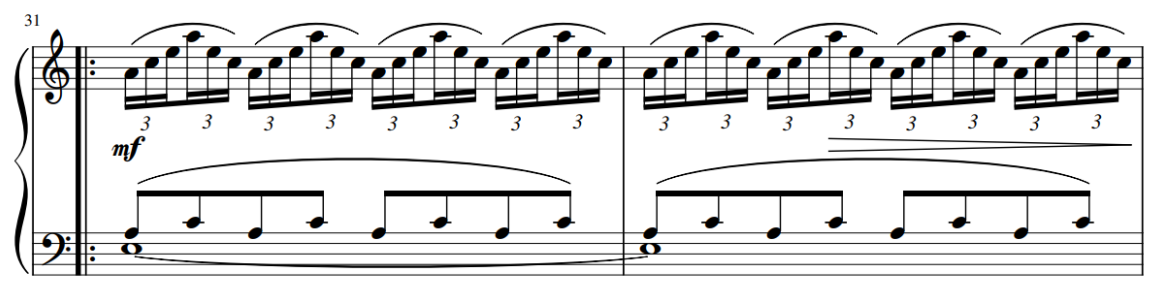

Ejemplo 1. Metamorphosis Two, cc. 31-32.

${ }^{2}$ Sus colaboraciones no se detendrán en The Thin Blue Line, sino que continuarán con $A$ Brief History of Time (1981) y The Fog of War: Eleven Lessons from the Life of Robert S. McNamara (2003). Además, Glass pondrá música a They Were There (2011), un corto documental sobre el centenario de la compañía IBM.

3 Si bien la primera aparición de esta obra se corresponde con el álbum Solo Piano (1989), la partitura no será editada hasta 1991 por Chester Music, reeditándose en 2006 por Wise Publications en la antología The Piano Collection. 


\section{Objetivos y metodología}

El objeto de este estudio es el de considerar el documental The Thin Blue Line (1988) bajo el prisma de los paralelismos pragmáticos que se pueden contemplar tanto en el trabajo del director Errol Morris como en el del compositor Philip Glass. En cuanto a este documental, se ha debatido ampliamente en torno a los procedimientos de Morris y su utilización de los reenactment "como técnica de investigación" (Fallon, 2012, p. 52), dejando a un lado los procedimientos musicales de Philip Glass. Éstos aparecen de forma somera a lo largo de gran parte de los estudios, refiriéndose únicamente a la música de Glass por sus cualidades hipnóticas y etéreas. Debido a la riqueza de aspectos que se pueden contemplar en este documental, proponemos un acercamiento a estos reenactments considerando también la función que la música desempeña en ellos.

Así, este trabajo trata de ahondar en el estudio de la música en el cine documental, dando la importancia que se merece -tal y como afirma Bill Nichols- "a la mitad audio del término audiovisual” (Nichols, 2016, p. 59). Para ello hemos requerido de una metodología interdisciplinar basada tanto en los estudios musicológicos como en los de cine documental, así como en aquellas publicaciones que abordan tanto la música en el cine como los usos de ésta en el cine documental. De este modo, seguimos la línea de estudios que abordan este tema tales como Music and Sound in Documentary Film, editado en 2016 por Holly Rogers o el monográfico sobre el uso de la música de Philip Glass en el cine -donde se cita su aparición en el cine documental pero no se contempla con detenimiento el caso de The Thin Blue Linepublicado en 2015 por Tristan Evans: Shared Meanings in the Film Music of Philip Glass: Music, Multimedia and Postminimalism. Por otro lado, para el análisis de los reenactments nos hemos servido principalmente de publicaciones sobre cine documental como Introducción al documental (2013) o Speaking Truth with Film: Evidence, Ethics, Politics in Documentary (2016), ambas de Bill Nichols o la editada por Jonathan Kahana en 2016, The Documentary Film Reader: History, Theory, Criticism. 
Además, se han utilizado tanto monográficos en torno a la figura de Morris como en relación con el compositor Philip Glass: nos referiremos a publicaciones como American Minimal Music. La Monte Young, Terry Riley, Steve Reich, Philip Glass (Mertens, 1983) y Palabras sin música (Glass, 2015), u otras como el caso de Three Documentary Filmmakers: Errol Morris, Ross McElwee, Jean Rouch (Plantinga, 2009) o The Cinema of Errol Morris (Resha, 2015).

\section{Reenactments y procesos de repetición minimalistas}

El peculiar uso de reenactments - "la recreación más o menos auténtica de sucesos anteriores"4 (Nichols, 2008, p. 72)- es probablemente uno de los aspectos más alabados y estudiados de The Thin Blue Line. Éstos desempeñan en gran medida el papel de motor estructural del documental, separando y complementando cada una de las versiones y variaciones que se dan sobre el asesinato, pasando a posicionarse como una herramienta de investigación, más que como un recurso estético de reconstrucción de los hechos. Tal y como afirma Bill Nichols, los reenactments fueron "materia prima del documental hasta que fueron masacrados por el cinéma-verité" 5 (Nichols, 2016, p. 34) por no comulgar con la espontaneidad y realidad que se presuponía en el cine documental. En la misma línea, el cinéma-verité tenía una opinión negativa sobre la aparición de la música no diegética -es decir, aquella que no es recogida por la cámara y que no se corresponde con el momento y el lugar en el que se desarrolla la escena- por ser vista como un elemento de postproducción y posicionarse en contra de la naturalidad del documental. De este modo, el propio Michel Brault afirmaba:

la música es una interpretación, es el cineasta quien dice: bien, voy a hacerte escuchar música aquí, sobre estas imágenes para crear una impresión concreta [...] [el documental] es realismo, y la música no tiene lugar (Rogers, 2015, pp. $2-3)^{6}$.

\footnotetext{
4 "Reenactments, the more or less authentic re-creation of prior events, [...]"

5 "[reenactments] were a staple of documentary representation until they were slain by the "verité boys" of the 1960s".

6 "Music is an interpretation, it's the filmmaker who says, alright I'm going to make you
} 
Así pues, esto conllevó que tanto la música no diegética como los reenactments se excluyesen de la estética del documental en busca de una mayor realidad y verosimilitud sin intermediación -en teoría- por parte del cineasta.

Errol Morris refutará magistralmente en The Thin Blue Line ambos presupuestos del cinéma-verité, utilizando el reenactment como herramienta de investigación y sirviéndose de la música de Philip Glass para generar una ambientación idónea para cada situación. Así, en este sentido, es interesante recordar las palabras del director, quien afirmaba que sus películas "se hacen -no completamente, pero en gran medida- en postproducción y edición”7 (Morris en: Millard 2014, p. 72), situándose en un planteamiento diametralmente opuesto al promulgado por sus predecesores del cinéma-verité. En 1988, el momento de publicación de este documental, los reenactments volvían a ser empleados de forma recurrente, pero no obstante ha de destacarse la labor de Errol Morris en su uso ya que como afirma David Resha: "mientras otros documentales podían usar reenactments, muy pocos aparecían tan pulidos o tan estéticamente maduros como los de The Thin Blue Line"8 (2015, p. 57). Esta afirmación emana tanto de la calidad como del detalle con los que se abordan las reconstrucciones. Un claro ejemplo es el ampliamente citado plano del batido arrojado por la agente de policía desde el coche. En él, Morris busca detenerse e incidir en los detalles: su lanzamiento, su caída y su relevancia como prueba del incoherente testimonio de la agente, ya que la trayectoria había finalizado en un punto distinto al que ella afirmaba.

Así, los reenactments en The Thin Blue Line son empleados por Morris principalmente con el afán de mostrar cómo "nuestra visión es parcial e incompleta"9 (Morris en: Millard 2014, p. 66), por este motivo el director no presenta en ningún momento la forma en la que sucedió el asesinato, sino repeticiones de acuerdo con los distintos testimonios. Esta continua

listen to music here on top of these images to create a certain impression [...] It's [documentary film] realism, and music has no place there".

7 "My movies are made - not entirely, but to a large degree - in postproduction and editing".

8 "while other documentaries might use reenactments, very few look as polished or aesthetically mature as those in The Thin Blue Line".

9 "Our vision is partial and incomplete". 
repetición del mismo hecho guarda una estrechísima vinculación con la técnica minimalista, y especialmente con la de Glass, quien por aquel entonces ya había comenzado a escribir un tipo de música "basada en la repetición y en ciclos rítmicos"10 (Mertens, 1983, p. 68).

$\mathrm{Si}$ reflexionamos sobre la repetición en la música minimalista podemos pensar, en la línea de las afirmaciones de Wim Mertens, que "en la música repetitiva, el concepto de obra se ha sustituido por la noción de proceso, ya no hay sonidos con mayor relevancia que otros"11 (1983, p. 88). De la misma manera, este presupuesto puede ser considerado en los reenactments. Ninguno de ellos es más real o cierto que cualquiera de los demás, ninguno muestra lo sucedido, sino que todos ellos se dirigen a la manifestación de la falsedad de los testimonios, son un mismo proceso hacia la construcción de la inocencia y consecuente libertad de Adams. Así, puede decirse que cada reenactment no constituye un pequeño fragmento de un reenactment total que se identificaría con la visión objetiva y definitiva de los hechos- sino que cada uno de ellos conforma distintas visiones, todas ellas señalando la construcción de la mentira que ha empañado la totalidad del proceso.

Por su parte, la música minimalista realiza un procedimiento similar en cuanto a las unidades temporales. Esta música se opone al clásico concepto occidental de la fragmentación de una unidad de tiempo superior -cuadrada o redonda- subdividida en unidades menores -negras, corcheas, semicorcheas, etc.- optando, sin embargo, por buscar la generación de unidades mayores a través de la síntesis de unidades menores. Así, la música se generaría a través de bucles o repeticiones (que serían los elementos mínimos) sobre las que se añaden los distintos materiales que darán forma a la obra. Este procedimiento se fundamenta en gran medida en la música tradicional de la India, con la que Philip Glass aparece especialmente vinculado a través de su relación con Ravi Shankar y de los numerosos viajes que ha realizado a la India a lo largo de su vida (Glass, 2015, pp. 166-174; 192-247; Mertens, 1983, pp. 67-68).

\footnotetext{
10 "Glass wrote music based on repetition and rhythmic cycles".

11 "one finds that in repetitive music the concept of work has been replaced by the notion of process, and that no one sound had any greater importance than any other".
} 
De este modo, podemos ver un primer paralelismo en los procedimientos empleados tanto por Errol Morris como por Philip Glass. Morris no establece una 'verdad' sobre el asesinato al inicio, pasando a mostrarla más detalladamente a lo largo del documental, sino que opta por reiterar las incongruencias entre las distintas versiones, construyendo así la falsedad de las acusaciones y por tanto la inocencia de Adams. Por su parte, Glass toma modelos musicales mínimos -como es el caso del motivo de tercera menorcon el fin de crear estructuras mayores a través de diversos procedimientos como la adición. Así, tanto Glass como Morris ofrecen una suerte de variaciones sobre un mismo tema, siendo el material base para el director la reconstrucción del asesinato y para el compositor el motivo de terceras.

\section{La ironía como proceso constructor de la inocencia: reenactments y modalidad}

Como hemos indicado, Morris empleará el reenactment como una herramienta de investigación a través de la cual ofrece al espectador cada una de las versiones de los testigos o participantes del asesinato. No obstante, ha de reiterarse que en ningún momento ofrecerá al espectador "lo que realmente pasó”, sino que cada reenactment se corresponderá con las distintas versiones de los hechos que se oyen a lo largo del documental, dando "fe de la nebulosa de dudas en las que estaba envuelta la verdad en torno a quién mató o no al policía” (Nichols, 2016, p. 72)12, a la par que se muestra la forma en la que la realidad "puede ser reflejada desde distintos puntos de vista"13 (Tugce Kaymaz, 2012, p. 76).

Atendiendo a la forma de utilización de éstos, Bill Nichols sitúa a los reenactments de The Thin Blue Line en la categoría de parodia e ironía, afirmando que en este documental "la perspectiva irónica se dirige más a la subjetividad de los entrevistados que a la capacidad de los reenactment para

12 "No single reenactment [...] represents "what really happened", but all attest to the cloud of doubt and self-deception that shrouded what was, in fact, the truth of who did or did not kill the fallen police officer".

${ }_{13}$ "this scene is showing that reality can be reflected from different points of view". 
capturar la autenticidad de los sucesos pasados” (Nichols, 2016, p. 47)14. Es decir, en cierto modo Morris realiza una suerte de comentario irónico en torno a la injusta condena de Adams mostrando una y otra vez la inconsistencia de las declaraciones que le llevaron al corredor de la muerte.

A nivel musical, Philip Glass establece de forma paralela un discurso irónico a lo largo del documental, especialmente en su comienzo. En los títulos iniciales Glass incorpora una variación del motivo de terceras menores del que hemos hablado con anterioridad. En este caso, la tonalidad pasa a ser Mi Mayor, con lo que el motivo pasa a fundamentarse en el intervalo de tercera mayor $\mathrm{Mi}$ - Sol\#, estando esta tonalidad tradicionalmente vinculada con sucesos triunfales y grandiosos: es decir, lo opuesto a los hechos acontecidos en The Thin Blue Line. Sin embargo, puede verse que el motivo de terceras menores no desaparece totalmente sigue apareciendo en la repetición en semicorcheas arpegiadas de las notas Sol\#-Si (véase Ejemplo 2).

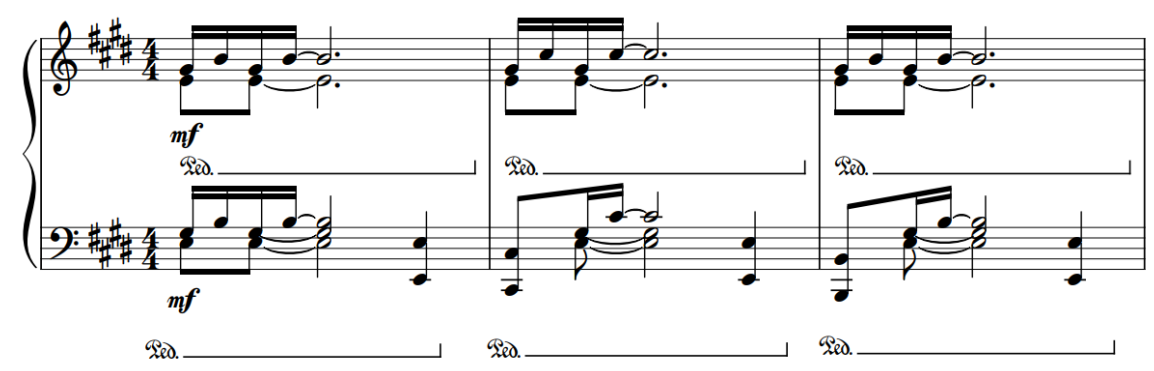

Ejemplo 2. Material inicial de Looking, cc. 1-3 (Glass, s. f.)

Inmediatamente después de estos títulos iniciales comienza a oírse un fragmento que en la grabación de la banda sonora (Glass, 2003) ${ }^{15}$ lleva por nombre Adam's Theme, el cual entra en conflicto con el material que se acaba de escuchar, ya que aparece en tono menor. Este nuevo fragmento musical en este caso cercano a Metamorphosis One puesto que el material se desarrolla casi de forma idéntica- aparece desde el inicio de las imágenes, con los diversos planos de la ciudad y se mantiene a lo largo de los primeros

\footnotetext{
14 "[...] his ironic perspective takes aim more at the subjectivity of his interviewees than at the capacity of the reenactment to capture the authenticity of past events".

${ }^{15}$ Hemos optado por referirnos a esta grabación y no a la de 1988 ya que en esta segunda no aparece la música aislada, sino que está conformada por la totalidad de la banda sonora del documental -entendida en sentido estricto- incluyendo así las entrevistas, reenactments, etc.
} 
testimonios de Randall Adams y David Harris, pasando rápidamente a asociarse con las intervenciones de Adams.

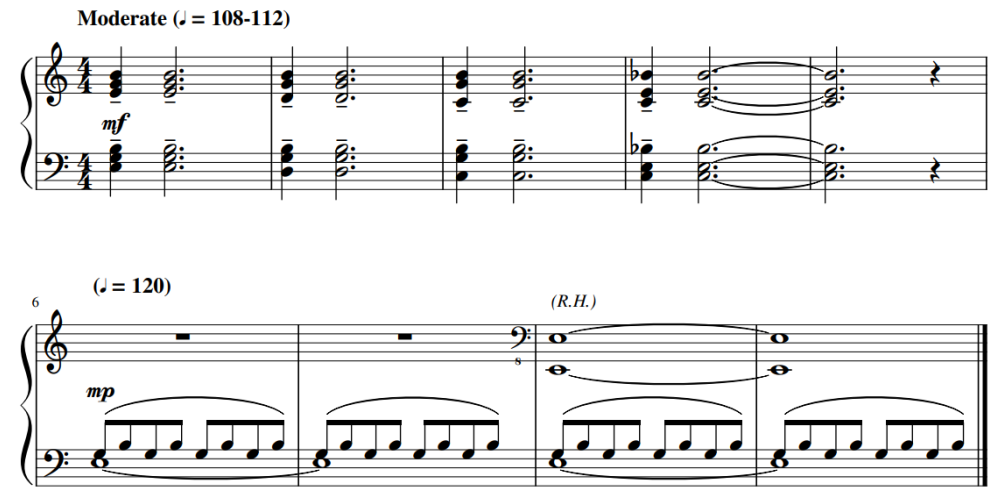

Ejemplo 3. Metamorphosis One, cc. 1-9.

Además de las incipientes terceras menores sobre las que se fundamenta el arpegio -que se desarrollarán a lo largo de todo el film- comienza a aparecer un motivo en la voz inferior, de carácter descendente y por grados conjuntos -es decir, en intervalos de tono o semitono- recurso que ya desde la retórica musical de finales del Renacimiento e inicios del Barroco se utilizaba para vincular palabra y música en momentos en los que el texto se refiriese a caídas, descensos, etc. En este caso, se vincula con un descenso hacia el infierno, una suerte de condena, la cual coincide con las impresiones que la madre de Adams tiene en torno a Dallas: "Hell on Earth”. Así, podemos ver un peculiar caso de catabasis una figura retórica que se es definida por Athanasius Kircher en 1650 como "una frase melódica descendente en relación con textos que hablan de descender, tierra, infierno, humillación y similares” (Suárez García, 2012, p. 283).

Por su parte, la reiteración -en este caso de las ya mencionadas, y casi omnipresentes, terceras menores- es un procedimiento recurrente a lo largo de toda la obra de Glass, cuya técnica compositiva se fundamenta "en una repetición, en la cual las figuras musicales son estructuradas de acuerdo a un método de adición [...] el rasgo más característico de su estilo"16 (Mertens, 1983, p. 67). Por su parte, la codificación de sentimientos de tristeza y

16 "Glass's more personal music is based on repetition in which the musical figures are structures according to an additive method, and this method of structuring is, without any doubt, the most characteristic feature of his style". 
desolación en el intervalo de tercera menor concuerda con el presupuesto de uno de los abanderados del movimiento de la música minimalista americana, La Monte Young, quien afirmaba que "cada intervalo determina un sentimiento distinto"17 (Mertens, 1983, p. 89). Asimismo, se comprueba la forma en la que Glass articula un "sentimiento de condena y desesperación [...] existe la oportunidad de encontrarse en una carretera y que sea el inicio de un viaje de cabeza hacia el Texas Department of Correction"18 (Bates y Morris, 2016, pp. 807-809).

En definitiva, Glass aprovecha las cargas emocionales del intervalo de tercera menor, al que añade un motivo descendente. Así consigue que ambos recursos musicales entre en confrontación con el primer material escuchado, construido sobre el intervalo de tercera mayor. De este modo, dicho contraste consigue que el fragmento en tono mayor comience a percibirse como un comentario irónico al 'triunfo de la Justicia' estadounidense, al verse opuesto al material en modo menor, que vaticina la injusticia. Además, estos compases en modo mayor no serán reutilizados en los créditos finales, tras la declaración de David Harris y el descubrimiento de la corrupción de la justicia, negando así la representación de la condena original como un hecho elogiable. En estos créditos finales, sin embargo, Glass empleará el pasaje sobre el que luego realizará su Metamorphosis Two (véase Ejemplo 1), concretamente el material en tresillos. Así, el momento de los créditos finales parece el más cercano a lo que podríamos denominar clímax sonoro del documental, situándose justo después de la declaración de culpabilidad de Harris que conlleva la inocencia de Adams y la resolución del caso. Como ya se ha indicado, ésta no es la única ocasión en la que Glass utiliza este motivo en tresillos de Metamorphosis Two para enfatizar el punto álgido de un film, siendo un ejemplo el momento en el que en Las Horas el personaje Richard Brown decide cometer suicidio dejándose caer por la ventana.

Esta 'ironía musical' a la que nos acabamos de referir participa de forma activa y fundamental en la construcción de la inocencia de Adams que Morris

17 "that each harmonic interval determines a distinct feeling".

18 "[Philip Glass' music] gives the film an underlying feeling of inexorability of inevitability [...] there's a chance meeting on a roadway and it's the beginning of a headlong trip to the Texas Department of Correction”. 
realiza a través de diversos elementos. La inocencia es enfatizada por el director a través de una serie de detalles en la representación del condenado: por ejemplo, desde el primer momento Randall Adams se nos muestra "bien afeitado y con camisa blanca, generando un efecto de tipo normal"19 (Curry, 1995, p. 156), en contraste con el uniforme de preso con el que se representa a Harris. El primero no muestra signos de actitud violenta o beligerante, sino que se pregunta constantemente por qué tuvo que estar en el lugar equivocado en un momento poco oportuno: "Why did I meet this kid? Why did I run out of gas? But it happened, it happened".

Ya que The Thin Blue Line no presenta un modelo de narración convencional en el que el espectador siga el desarrollo a través de una voice-over (Tugce Kaymaz, 2012, p. 75), esta función es realizada por la música. La música que se vincula a Adams transmite señales de inocencia a través del énfasis en el modo menor y en el descenso por grados conjuntos, que se opone al tono mayor del triunfo de la Justicia, lo que en cierto modo presupone sonoramente el fatídico error cometido en la condena de Adams. Esto es confirmado por el material musical de los créditos finales a los que nos hemos referido.

Sin embargo, probablemente la raíz de la unión entre imagen y sonido en la intencionalidad de la construcción de la inocencia de Adams se halla en uno de los presupuestos fundamentales de la música minimalista: en ésta, "la percepción es parte integral y creativa del proceso, ya que el oyente no percibe un trabajo finalizado, sino que participa en su construcción" 20 (Mertens, 1983, p. 90). Esto se produce a través de las infinitas repeticiones de la música minimalista, que imposibilitan la capacidad del oyente para "mantener una atención constante"21 (Mertens, 1983, p. 81), transmitiendo la sensación "de que sólo escucha un fragmento de un continuum musical permanente"22 (Mertens, 1983, p. 71). Con este pretexto, puede entenderse la

19 "He looks well shaven and wears a white shirt; the overall effect is one of "averageness"-a clean-cut average person".

20 "In repetitive music perception is an integral and creative part of the musical process since the listener no longer perceives a finished work but actively participates in its construction".

${ }_{21}^{21}$ "[...] because of the long duration, it is impossible for the spectator to sustain a consistent attention".

${ }_{22}$ "[...] gives the listener the feeling that he or she only hears a fragment in a permanent 
inocencia de Adams no como un atributo que se le otorgue tras la declaración de culpabilidad de David Harris con la que se concluye el film, sino como un proceso por el que el espectador se mueve paulatinamente a lo largo del documental, guiado por las continuas formas de representación que tanto Morris como Glass realizan en conjunto a través de medios audiovisuales.

\section{Conclusiones}

Tal y como hemos visto, ciertos procedimientos creativos empleados por Errol Morris y Philip Glass pueden ser vistos como la expresión de conceptos similares en medios artísticos diferentes. En primer lugar, destaca la noción de repetición empleada por ambos: mientras Morris repite una y otra vez el reenactment del asesinato, Philip Glass crea una música sustentada en elementos "mínimos" repetitivos y cíclicos. Ambos, los reenactments y la técnica de repetición del minimalismo, pueden verse más como un proceso que como el resultado final y depurado de una obra concluida, un procedimiento similar al de la construcción de inocencia de Randall Adams que hace Morris. Asimismo, tanto Morris como Glass rechazan un procedimiento deductivo: negándose a representar reenactments que constituyan una recreación 'verdadera' de los hechos, o bien no derivando figuras rítmicas a través de la subdivisión de figuras mayores. Ambos optan por un proceso inductivo: Morris compone la falsedad de los testimonios a través de la unión de los reenactments, Glass establece grandes secciones musicales a través de la adición y repetición de intervalos de tercera menor. -

Por otro lado, tanto Morris como Glass utilizan la ironía como medio para construir la inocencia de Randall Adams. Glass alterna secciones en modo mayor y menor, vinculando el modo de Mi mayor del triunfo de la justicia en los títulos de inicio con el homónimo menor en la declaración de un Adams condenado de por vida, y cerrando el documental con unos créditos también en modo menor -un material musical que servirá a Glass como embrión de lo que será su obra Metamorphosis Two: éstos ya no pueden expresar ese 'triunfo' de la justicia del modo mayor tras la confesión de culpabilidad de 
Harris. Por su parte, Errol Morris utiliza los reenactments como una suerte de comentario irónico a los testimonios, mostrando visualmente su incoherencia y la falsedad de la condena de Adams. En definitiva, el presente trabajo ha tratado de aportar una nueva morada sobre el documental The Thin Blue Line, tratando de relacionar los procesos del cineasta Errol Morris con los del compositor Philip Glass para, de este modo, enfatizar la estrecha relación conceptual que pueden llegar a guardar.

\section{Referencias bibliográficas}

Algar, N. (1989). Errol Morris - Believe It or Not. Monthly Film Bulletin, $56(663)$.

Bates, P., y Morris, E. (2016). Truth not guaranteed. An Interview with Errol Morris (1989). En J. Kahana (Ed.), The documentary film reader: history, theory, criticism (pp. 807-809). New York: Oxford University Press.

Brooke, M. (2014). Endings...: The Thin Blue Line. Sight \& Sound, XXIV(9), 128.

Curry, R. (1995). Errol Morris' Construction of Innocence in The Thin Blue Line. Rocky Mountain Review of Language and Literature, 49(2), 153-167.

Evans, T. (2015). Shared Meanings in the Film Music of Philip Glass: Music, Multimedia and Postminimalism. Farnham: Ashgate.

Fallon, K. (2012). Several sides of Errol Morris. Film Quarterly, LXV(4), 4853 .

Glass, P. (s. f.). LOOKING - from «The Thin Blue Line». (B. Pittman, Ed.). Recuperado https://musescore.com/user/10536501/scores/5500385

de

Glass, P. (1989). Solo Piano [Disco]. CBS.

Glass, P. (2003). Music From The Thin Blue Line [Disco]. Orange Mountain Music.

Glass, P. (2006). The Piano Collection. London: Wise Publications.

Glass, P. (2015). Palabras sin música. (M. López, Trad.). Barcelona: Malpaso.

Kahana, J. (2016). Introduction to Section VI. En J. Kahana (Ed.), The documentary film reader: history, theory, criticism (pp. 723-725). New York: Oxford University Press. 
Mertens, W. (1983). American Minimal Music. La Monte Young, Terry Riley, Steve Reich, Philip Glass. (J. Hautekiet, Trad.). London: Kahn \& Averill.

Millard, K. (2014). 14 Lessons on Screenwriting from Errol Morris. En Screenwriting in a Digital Era (pp. 63-76). New York: Palgrave Macmillan.

Nichols, B. (2008). Reenactment and the Fantasmatic Subject. Critical Inquiry, 35(1), 72-89.

Nichols, B. (2013). Introducción al documental. (M. B. García, Trad.). Coyoacán: Universidad Nacional Autónoma de México.

Nichols, B. (2016). Speaking truth with film: evidence, ethics, politics in documentary. Oakland: University of California Press.

Plantinga, C. (2009). The Philosophy of Errol Morris. Ten Lessons. En W. Rothman (Ed.), Three documentary filmmakers: Errol Morris, Ross McElwee, Jean Rouch (pp. 43-59). Albany: State University of New York Press.

Resha, D. (2015). The Thin Blue Line. En The Cinema of Errol Morris (pp. 49-81). Middletown: Wesleyan University Press.

Rogers, H. (2015). Introduction. Music, Sound and the Nonfiction Aesthetic. En H. Rogers (Ed.), Music and Sound in Documentary Film (pp. 119). New York: Routledge.

Suárez García, J. I. (2012). El embellecimiento retórico en el Officium Defunctorum de Tomás de Victoria. Revista de Musicología, XXXV(1), 263-294.

Tugce Kaymaz, Ö. (2012). The Thin Blue Line: How can we destroy actuality with editing? Cinej Cinema Journal, 1(2), 73-78. 